\title{
Níveis séricos de zinco materno e associação com a pré-eclampsia - Uma revisão
}

\section{sistemática}

\author{
Maternal zinc serum levels and association with pre-eclampsia - A systematic review \\ Niveles en suero de zinc materno y asociación con pre-eclampsia - Una revisión sistemática
}

Ana Clara do Nascimento Borges ORCID: https://orcid.org/0000-0003-3599-380X

Universidade Federal do Piauí, Brasil

E-mail: claraborges25@gmail.com

Beatriz Gabrielle Silva Oliveira

ORCID: https://orcid.org/0000-0001-5631-7865

Universidade Federal do Piaú, Brasil

E-mail: bgs.oliveira@outlook.com

Ana Adélya Alves Costa

ORCID: https://orcid.org/0000-0003-1793-5307

Universidade Federal do Piauí, Brasil

E-mail: anaadelyaa@gmail.com

Lara Laiany Aguiar Souza

ORCID: https://orcid.org/0000-0002-4642-221

Centro Universitário Estácio do Ceará, Brasil

E-mail: laraaguiar10@ hotmail.com

Nara Vanessa dos Anjos Barros

ORCID: https://orcid.org/0000-0003-2044-7064

Universidade Federal do Piauí, Brasil

E-mail: naranessa@ufpi.edu.br

Diêgo de Oliveira Lima

ORCID: https://orcid.org/0000-0001-8211-9416 Universidade Federal do Piauí, Brasil E-mail: di.oliveiralima@ hotmail.com

Maria Lúcia de Moura Rocha Barbosa

ORCID: https://orcid.org/0000-0002-7633-9815 Universidade Federal do Piauí, Brasil E-mail: mlesperanca@gmail.com

Mariana Gosmão de Carvalho

ORCID: https://orcid.org/0000-0003-2793-7744 Universidade Federal do Piaú, Brasil E-mail: marygosmao92@gmail.com

Luana da Silva Amorim

ORCID: https://orcid.org/0000-0001-7506-9334 Universidade Federal do Piauí, Brasil E-mail: camilab10424@gmail.com

Francisca Camila Batista Lima

ORCID: https://orcid.org/0000-0002-9798-7029 Universidade Federal do Piauí, Brasil E-mail: camilab10424@gmail.com

\begin{abstract}
Resumo
Objetivo: Realizar um estudo no intuito de unir evidencias sobre a associação entre os níveis séricos de Zn materno e a ocorrência de pré-eclâmpsia em gestantes. Metodologia: Trata-se de uma revisão de literatura do tipo sistemática, a busca de artigos científicos foi realizada por dois autores de forma independente, entre os meses de Maio e Junho de 2021, nas seguintes bases de dados eletrônicas: National Library of Medicine (PubMed), Scopus e Science Direct. Utilizando como descritores controlados identificados nos Descritores em Ciência da Saúde (DECs) a seguinte combinação em inglês: ("Zinc AND pre-eclampsia”). Após a estratégia de busca dos artigos, foi realizado uma leitura dos títulos, após dos resumos e, por último, do texto completo para construção da tabela de resultados. Resultados: Foram encontrados um total de 1088 artigos por meio da busca nas bases de dados, após o processo de triagem, resultou em total de 11 artigos escolhidos para mensuração dos resultados, estes se tratavam de estudos caso-controle, estudo transversal comparativo, estudo transversal analítico e coorte prospectiva, publicados nos anos de 2016 a 2021 , sendo realizados com mulheres com PE e normotensas, com idade acima de 17 anos. Conclusão: Uma maioria dos estudos encontraram uma relação entre o baixo nível de zinco e a pré-eclâmpsia, deixado a entender que haja uma
\end{abstract}


correlação entre ambos, porém os estudos ainda são conflitantes, uma vez que alguns autores não encontraram essa associação. No entanto, é necessário a realização de estudos mais exatos sobre a temática para melhor entendimento. Palavras-chave: Zinco; Pré-eclâmpsia; Gestação.

\begin{abstract}
Objective: To carry out a study to compile evidence on the association between the serum levels of maternal $\mathrm{Zn}$ and the occurrence of preeclampsia in pregnant women. Methodology: This is a systematic review of the literature, the search for scientific articles was carried out by the authors independently, between May and June 2021, in the following electronic databases: National Library of Medicine (PubMed), Scopus and Science Direct. Using as controlled descriptors identified in the Descriptors of Health Sciences (DECs) the following combination in English: ("Zinc AND pre-eclampsia"). After the article search strategy, the titles were read, after the summaries and, finally, the full text to build the results table. Results: A total of 1088 articles were found through the search in the databases, after the screening process, it resulted in a total of 11 articles chosen for measuring the results, these were case-control studies, comparative cross-sectional study, cross-sectional analytical study and prospective cohort, published from 2016 to 2021, being carried out with women with PE and normotensive, aged over 17 years. Conclusion: The majority of the studies found a relationship between the low levels of zinc and the preeclampsia, understanding that there is a correlation between them, but the studies are contradictory, and that some authors have not found this association. Without embargo, it is necessary to carry out more accurate studies on the subject for a better understanding.
\end{abstract}

Keywords: Zinc; Pre-eclampsia; Gestation.

\title{
Resumen
}

Objetivo: Realizar un estudio para recopilar evidencia sobre la asociación entre los niveles séricos de Zn materno y la ocurrencia de preeclampsia en mujeres embarazadas. Metodología: Se trata de una revisión sistemática de la literatura, la búsqueda de artículos científicos fue realizada por dos autores de forma independiente, entre los meses de mayo y junio de 2021, en las siguientes bases de datos electrónicas: National Library of Medicine (PubMed), Scopus y Science Direct. Utilizando como descriptores controlados identificados en los Descriptores de Ciencias de la Salud (DECs) la siguiente combinación en inglés: ("Zinc AND pre-eclampsia”). Después de la estrategia de búsqueda de artículos, se leyeron los títulos, después de los resúmenes y, finalmente, el texto completo para construir la tabla de resultados. Resultados: Se encontraron un total de 1088 artículos a través de la búsqueda en las bases de datos, luego del proceso de cribado resultó en un total de 11 artículos elegidos para medir los resultados, estos fueron estudios de casos y controles, estudio transversal comparativo, transversal analítico estudio y cohorte prospectiva, publicado de 2016 a 2021, realizado con mujeres con EP y normotenso, mayores de 17 años. Conclusión: La mayoría de los estudios encontró una relación entre los niveles bajos de zinc y la preeclampsia, dejando entendido que existe una correlación entre los dos, pero los estudios aún son contradictorios, ya que algunos autores no encontraron esta asociación. Sin embargo, es necesario realizar estudios más precisos sobre el tema para una mejor comprensión.

Palabras clave: Zinc; Preeclampsia; Gestación.

\section{Introdução}

O zinco ( $\mathrm{Zn}$ ) é considerado um mineral essencial ao metabolismo, desempenhando um papel vital em diversos processos biológicos e estando presente em várias funções relacionadas ao corpo humano, sendo cofator de mais de 300 enzimas (Yuasuda, 2016). O Zn é o segundo oligoelemento mais abundante no organismo humano, encontrando-se em todos os tecidos do corpo e desempenhando funções cruciais na divisão celular, no metabolismo, no desenvolvimento sexual, na imunidade e na capacidade cognitiva, exercendo importantes ações estruturais, catalíticas e regulatórias (Chasapis, 2012).

Além dos aspectos abordados, o mineral referido também está presente no metabolismo energético, metabolismo de hidratos de carbono e de lipídios, síntese proteica, metabolismo de DNA e RNA, sendo essencial para a diferenciação e divisão celular, além do bom funcionamento do sistema imunológico (Grieger, 2015). O Zn participa de vários períodos de crescimento, como infância, puberdade e na gestação. No último período citado, o Zn é essencial para o bom crescimento e desenvolvimento embrionário e fetal, e no trabalho de parto (Cominetti, 2020).

Durante a gestação e lactação, as necessidades de Zn aumentam, em função de uma maior demanda para a embriogênese normal, crescimento fetal e secreção do leite (Prado et al, 2015). A exigência de Zn durante o terceiro trimestre de gestação é aproximadamente duas vezes mais elevada que a de mulheres não grávidas. Concentrações plasmáticas de Zn diminuem à medida que a gestação avança. Entretanto, uma análise revelou que a ingestão dietética média das gestantes não supera mais que 50\% da necessidade diária deste micronutriente (Mistry, Kurlack \& Young, 2014). 
A deficiência de Zn pode ocorrer como resultado de ingestão inadequada, absorção reduzida, aumento das perdas ou aumento da demanda desse mineral. Os grupos populacionais com maior risco de desenvolver deficiência de Zn são aqueles com maiores exigências fisiológicas, como mulheres grávidas e lactentes (Thomas et al., 2013). É comum a deficiência de micronutrientes em mulheres grávidas, contudo, a deficiência do mineral em questão tem sido associada a algumas patologias, entre elas, a pré-eclâmpsia (PE). Uma condição multissistêmica que ocorre após a $20^{\circ}$ semana gestacional, clinicamente caracterizada por proteinúria e hipertensão, atualmente a PE é considerada uma das principais causas de morbidade e mortalidade materna e pré-natal (Sanchez-Aranguera, 2014).

Muitos estudos buscaram a associação entre os níveis de Zn na PE, no entanto, alguns deles ainda se mostraram inconsistentes, alguns demonstraram níveis significativamente mais baixos de Zn em mulheres com PE em comparação com mulheres grávidas normotensas (Kanagal, 2014; Ikaraoha, 2016; Mohamed, 2019). Já outras pesquisas não encontraram diferenças significativas entre os dois grupos (Elmugabil, 2016; Enebe, 2020). Portanto, a presente revisão sistemática foi elaborada no intuito de reunir evidências sobre a associação entre os níveis séricos de Zn materno e a ocorrência de PE em gestantes.

\section{Metodologia}

O presente artigo trata-se de uma revisão sistemática de estudos presente na literatura científica do tipo qualitativa. Esse tipo de pesquisa foca em um determinado assunto, visando identificar, selecionar, avaliar e sintetizar as principais evidências disponíveis relacionadas ao tema escolhido (Galvão; Pereira,2014). O desenvolvimento do estudo obedeceu às seguintes etapas pré-estabelecidas: escolha do tema abordado para formulação da questão de pesquisa, busca dos descritores para posterior análise, escolha das bases de dados a serem extraídos os estudos, seleção dos critérios de elegibilidade, definição das informações a serem extraídas dos mesmos, avaliação dos estudos incluídos na revisão sistemática, interpretação dos resultados e exposição da revisão.

Para a construção da pergunta norteadora, utilizou-se a estratégia PICO (Santos, 2007), com "P" correspondente à população (gestantes); "I" à observação (observação dos estudos que analisaram níveis de $\mathrm{Zn}$ ), "C" à comparação (gestantes com PE versus normotensas); "O” correspondendo ao desfecho (Qualidade da gestação), que resultou no questionamento a seguir: Há relação entre o nível sérico de Zn materno com casos de PE gestacional?

Utilizou-se como descritores controlados identificados nos Descritores em Ciência da Saúde (DECs) a seguinte combinação em inglês: ("Zinc AND pre-eclampsia"). A busca de artigos científicos foi realizada por dois autores de forma independente, entre os meses de Maio e Junho de 2021, nas seguintes bases de dados eletrônicas: National Library of Medicine (PubMed), Scopus e Science Direct.

Os estudos escolhidos para compor o trabalho foram aqueles que obedeceram aos critérios de elegibilidade, cuja temática respondesse à pergunta norteadora, que foram: estudos originais disponíveis na íntegra, estudos longitudinais, transversais, estudo de coorte, casos controle e estudo em animais, publicados nos últimos 5 anos (2016 - 2021), sem restrição de idade ou etnia para os participantes. Foram excluídos da pesquisa estudos que não tinha seu conteúdo completo disponível, estudos de revisão, estudos de caso, relatos de experiência, casos clínicos, teses, dissertações e capítulos de livro (Figura 1).

Após a estratégia de busca dos artigos, foi realizado uma leitura dos títulos, após dos resumos e, por último, do texto completo para construção da tabela de resultados, de forma que os passos foram seguidos pelos dois autores de forma independente. 
Figura 1. Fluxograma dos estudos avaliados para elegibilidade e incluídos na revisão

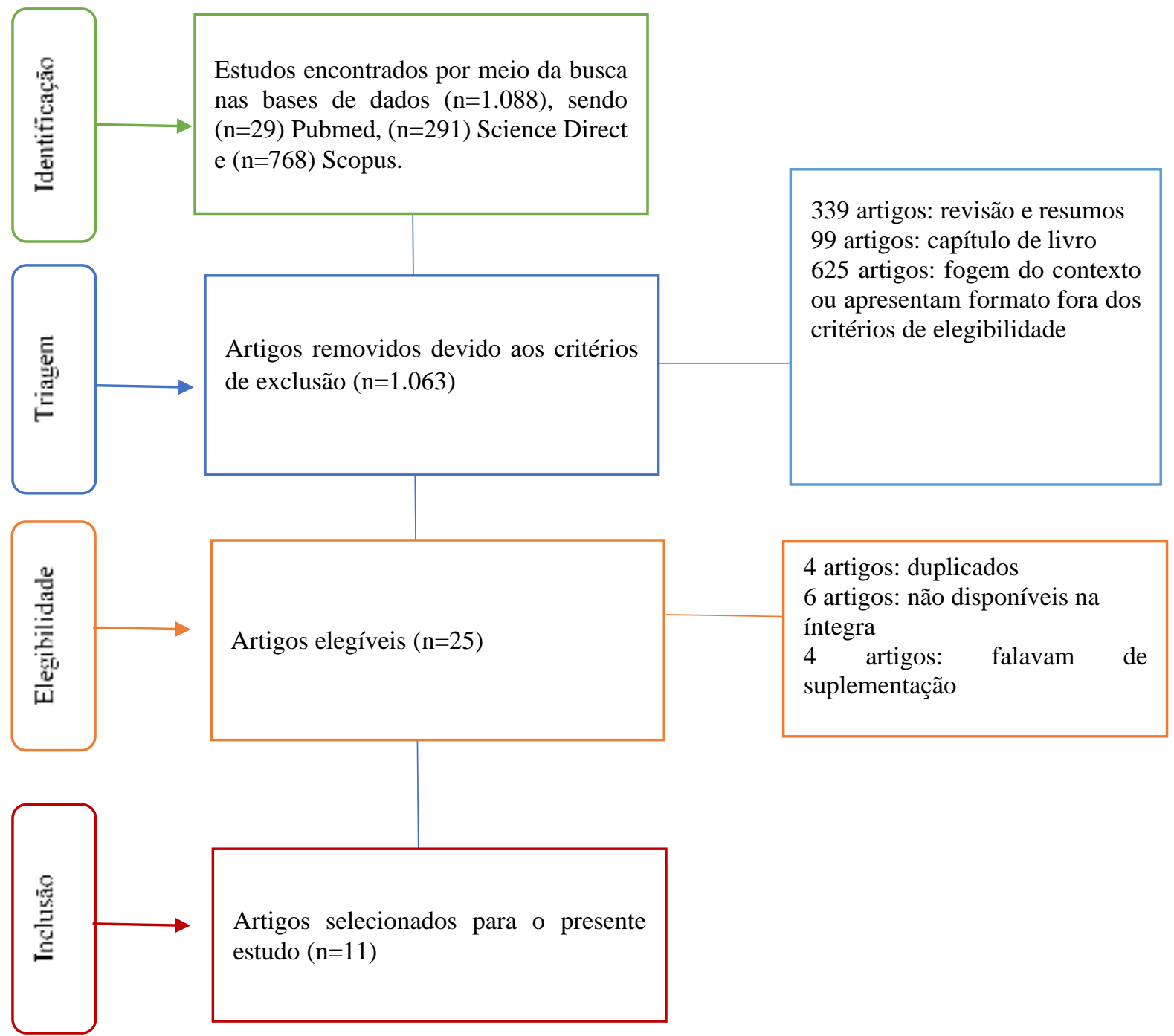

Fonte: Autores.

Para construção e melhor compreensão dos resultados foram selecionadas informações dos artigos elegíveis, como nome dos autores e ano de publicação, objetivos do estudo, número e idade dos participantes do estudo, tipo de estudo, métodos utilizados na construção dos mesmos e principais resultados. Para realização desta revisão, as recomendações PRISMA foram seguidas (Galvão, Pansani \& Harrad, 2015).

\section{Resultados}

Foram encontrados um total de 1088 artigos por meio da busca nas bases de dados escolhidas. Após o processo de triagem, foram retirados os artigos que estavam duplicados e indisponíveis na íntegra (n=9) e os que abordavam resultados sobre suplementação de $\mathrm{Zn}(\mathrm{n}=4)$, que não é o foco da pesquisa. Nesta revisão, foram utilizados 11 artigos, no qual o Quadro 1 mostra as características dos estudos incluídos

A partir da avaliação dos estudos selecionados, observou-se que estes se tratavam de estudos caso-controle, estudo transversal comparativo, estudo transversal analítico e coorte prospectiva, publicados nos anos de 2016 a 2021 , sendo realizados com mulheres com PE e normotensas, com idade acima de 17 anos. Com relação às análises do elemento mineral Zn, em 9 estudos foram coletadas amostras de sangue para analisar as concentrações de Zn e outros micronutrientes, 1 deles 
também coletou amostras de cabelo da região púbica, e 2 estudos analisaram os níveis de $\mathrm{Zn}$ por meio de dados obtidos do consumo alimentar relatado pelas gestantes.

Segundo Keshavarz et al. (2017), os níveis de Zn estavam baixos em mulheres com PE $(0,69 \pm 0,21)$ quando comparados com o grupo controle $(0,87 \pm 0,30)$, apresentando diferença estatisticamente significativa $(\mathrm{p}<0,001)$. Assim como no estudo de Mazloomi et al. (2020); Shaikh et al. (2017) e Jamal et al. (2017) que também verificaram uma diminuição dos níveis de Zn em gestantes com PE.

Oliveira et al. (2016) e Gul et al. (2021) demonstram em seu estudo que os níveis séricos de Zn se encontraram baixos nos grupos de gestantes saudáveis e no grupo com PE, sem diferença significativa entre os dois grupos (p>0,05). Enebe et al. (2020) também não encontraram diferença estatística significativa, mostrando que a concentração dos níveis de Zn não teve variação entre os dois grupos. 
Quadro 1. Estudos utilizados para construção dos resultados.

\begin{tabular}{|c|c|c|c|c|c|}
\hline $\begin{array}{l}\text { Autor } \\
\text { (ano) }\end{array}$ & Objetivos & $\begin{array}{l}\text { Participantes (n) } \\
\text { e idade (anos) }\end{array}$ & $\begin{array}{ll}\text { Tipo } & \text { de } \\
\text { estudo } & \\
\end{array}$ & Metodologia & Resultado \\
\hline $\begin{array}{l}\text { Oliveira et } \\
\text { al. }(2016)\end{array}$ & $\begin{array}{l}\text { Avaliar a ingestão de } \\
\text { nutrientes e coeficientes de } \\
\text { variação em gestantes com } \\
\text { PE. }\end{array}$ & $\begin{array}{l}178 \text { gestantes }= \\
89 \text { diagnosticadas } \\
\text { com PE e } 89 \\
\text { saudáveis. Idade } \\
\text { média de } 25,8 \\
\text { anos. }\end{array}$ & $\begin{array}{l}\text { Caso } \\
\text { controle. }\end{array}$ & $\begin{array}{l}\text { Análise de dados } \\
\text { socioeconômicos, } \\
\text { histórico de PE e } \\
\text { familiares, } \\
\text { antropométricos, hábitos } \\
\text { alimentares e ingestão de } \\
\text { nutrientes. }\end{array}$ & $\begin{array}{l}\text { Os níveis séricos de } \mathrm{Zn} \text { se mostraram reduzidos em ambos os } \\
\text { grupos sem diferença significativa entre os grupos }(\mathrm{p}=0,123) \text {. } \\
\text { Ingestão média de zinco por gestantes com pré-eclâmpsia } \\
(6,3 \pm 2,6) \text { e em gestantes sem pré-eclâmpsia }(7,2 \pm 5,9) \text {. }\end{array}$ \\
\hline $\begin{array}{l}\text { Elmugabil } \\
\text { et al. } \\
(2016)\end{array}$ & $\begin{array}{l}\text { Investigar os níveis de } \mathrm{Ca} \text {, } \\
\mathrm{Mg}, \mathrm{Zn} \text { e } \mathrm{Cu} \text { em mulheres } \\
\text { com } \mathrm{PE} \text {. }\end{array}$ & $\begin{array}{l}100 \text { participantes } \\
(50 \text { sem PE e } 50 \\
\text { com PE. Idade } \\
\text { média de } 28,6 \\
\text { anos. }\end{array}$ & $\begin{array}{l}\text { Caso } \\
\text { controle. }\end{array}$ & $\begin{array}{l}\text { Coletas de dados } \\
\text { antropométricos (peso, } \\
\text { altura e IMC) e analise dos } \\
\text { níveis séricos de } \mathrm{Ca}, \mathrm{Mg} \text {, } \\
\mathrm{Cu} \text { e Zn. }\end{array}$ & $\begin{array}{l}\text { Não houve diferenças significativas nos níveis de } \mathrm{Zn}, \mathrm{Cu} \text { e } \mathrm{Zn} \\
\text { /proporção de } \mathrm{Cu} \text { entre mulheres com PE }(108,0 \\
(91,6-131,7)) \text { e os controles }(102,0(82,8-124,0)(\mathrm{p}=0,254) \text {. }\end{array}$ \\
\hline $\begin{array}{l}\text { Latif et al., } \\
2016\end{array}$ & $\begin{array}{l}\text { Verificar o nível sérico de } \\
\text { Zn em gestantes saudáveis } \\
\text { e gestantes com PE. }\end{array}$ & 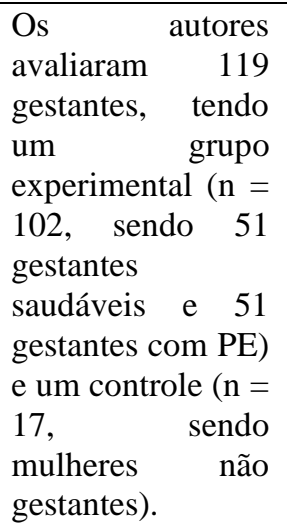 & $\begin{array}{l}\text { Foi realizado } \\
\text { um estudo } \\
\text { caso- } \\
\text { controle. }\end{array}$ & $\begin{array}{l}\text { Coleta sanguínea de todas } \\
\text { as participantes para } \\
\text { avaliar a concentração de } \\
\text { Zn. }\end{array}$ & $\begin{array}{l}\text { A média dos níveis séricos de } \mathrm{Zn} \text { no grupo experimental no } 1^{\circ} \\
\text { trimestre foi de } 0,78 \pm 0,25 \mathrm{mg} / \mathrm{l} \text {, no } 2^{\circ} \text { trimestre foi } 0,53 \pm \\
0,09 \mathrm{mg} / \mathrm{L} \text { e no } 3^{\circ} \text { trimestre foi } 0,56 \pm 0,08 \mathrm{mg} / \mathrm{l} \text {. Enquanto a } \\
\text { média encontrada de } \mathrm{Zn} \text { sérico no grupo controle (não } \\
\text { gestante) } 0,61 \pm 0,12 \mathrm{mg} / \mathrm{L} \text {, dado que não mostrou ter } \\
\text { diferença significativa }(\mathrm{p}>0,05) \text { da concentração sérica de } \mathrm{Zn} \\
\text { em mulheres com PE. A média dos níveis séricos de } \mathrm{Zn} \text { em } \\
\text { gestantes saudáveis foi de } 1,44 \pm 0,14 \mathrm{mg} / \mathrm{L} \text { em comparação } \\
\text { com o grupo de gestantes com } \mathrm{PE} \text {, que foi de } 0,25 \pm 0,02 \\
\text { mg/L, diferença estatisticamente significativa entre os grupos } \\
(\mathrm{p}<0,05) \text {. }\end{array}$ \\
\hline $\begin{array}{l}\text { Keshavarz } \\
\text { et al. } \\
(2017)\end{array}$ & $\begin{array}{l}\text { Avaliar o status do perfil } \\
\text { lipídico e os sistemas } \\
\text { antioxidantes em PE } \\
\text { medindo os níveis de Zn e } \\
\text { Cu e as atividades de SOD. }\end{array}$ & $\begin{array}{l}2 \text { grupos com } 100 \\
\text { mulheres } \\
\text { diagnosticadas } \\
\text { com PE e } 100 \\
\text { caso controle. } \\
\text { Idade entre } 16-42 \\
\text { anos. }\end{array}$ & $\begin{array}{l}\text { Caso } \\
\text { controle. }\end{array}$ & $\begin{array}{l}\text { Coleta de parâmetros } \\
\text { antropométricos (peso, } \\
\text { altura, e IMC), e análise } \\
\text { de níveis plasmáticos de } \\
\text { Zn e Cu e atividade da } \\
\text { enzima antioxidante. }\end{array}$ & $\begin{array}{l}\text { Baixos níveis plasmáticos de zinco em mulheres grávidas com } \\
\text { pré-eclâmpsia }(0,69 \pm 0,21) \text { em comparação com o grupo } \\
\text { controle }(0,87 \pm 0,30) \text {, nível de significância }(p<0,001) \text {. }\end{array}$ \\
\hline Shaikh et & Comparar & gestantes, & Estudo & Foram coletadas amostras & Os níveis séricos de $\mathrm{Zn}$ em mulheres normotensas foram \\
\hline
\end{tabular}




\begin{tabular}{|c|c|c|c|c|c|}
\hline al. (2017) & $\begin{array}{l}\text { plasmáticos de CoQ10, Zn, } \\
\text { Cu e Mg em gestantes } \\
\text { saudáveis e em mulheres } \\
\text { com PE. }\end{array}$ & $\begin{array}{l}\text { com idade entre } \\
20 \text { e } 30 \text { anos. }\end{array}$ & $\begin{array}{l}\text { transversal } \\
\text { comparativo. }\end{array}$ & $\begin{array}{l}\text { de sangue das voluntárias } \\
\text { para analisar os níveis } \\
\text { plasmáticos de CoQ10, } \\
\mathrm{Zn}, \mathrm{Cu} \text { e } \mathrm{Mg} \text {. }\end{array}$ & $\begin{array}{l}\text { anotados como }(5,09 \pm 0,29) \mathrm{mg} / \mathrm{dL} \text { em comparação com } \\
\text { mulheres com PE com } 2,94 \pm 0,44 \mathrm{mg} / \mathrm{dL} \text {. A concentração } \\
\text { sérica de } \mathrm{Zn} \text { reduziu no grupo caso em comparação com os } \\
\text { indivíduos de controle com diferença significativa }(\mathrm{p}<0,05) \text {. }\end{array}$ \\
\hline $\begin{array}{l}\text { Jamal et al. } \\
\text { (2017) }\end{array}$ & $\begin{array}{l}\text { O objetivo deste estudo é } \\
\text { prevenir PE em mães e seu } \\
\text { recém-nascido. }\end{array}$ & $\begin{array}{l}\text { Um } \quad \text { grupo } \\
\text { controle }(\mathrm{n}=40) \\
\text { e um grupo caso } \\
(\mathrm{n}=40) \text { com a } \\
\text { idade entre } 17 \text { e } \\
46 \text { anos. }\end{array}$ & $\begin{array}{l}\text { Estudo } \\
\text { transversal e } \\
\text { comparativo. }\end{array}$ & $\begin{array}{l}\text { Houve a coleta do } \\
\text { histórico de obstetrícia, } \\
\text { avaliação de antropometria } \\
\text { e dados clínicos. Foi feita } \\
\text { a coleta de sangue para } \\
\text { analisar } \mathrm{Zn}, \mathrm{Cu} \text { e Mg. }\end{array}$ & $\begin{array}{l}\text { O valor médio dos níveis séricos de } \mathrm{Zn} \text { no grupo caso era de } \\
2,94 \pm 0,45 \mathrm{mg} / \mathrm{dL} \text {, enquanto a média do grupo controle foi } \\
5,11 \pm 0,21 \mathrm{mg} / \mathrm{dL}, \text { a diferença foi estatisticamente } \\
\text { significativa }(\mathrm{p}<0,05) \text {. }\end{array}$ \\
\hline $\begin{array}{l}\text { Maduray } \\
\text { et al. } \\
(2017)\end{array}$ & $\begin{array}{l}\text { Foi avaliar } r \\
\text { concentrações de treze } \\
\text { diferentes elementos em } \\
\text { amostras de cabelo e soro } \\
\text { de mulheres com um } \\
\text { diagnóstico de PE. }\end{array}$ & $\begin{array}{l}\text { Um grupo } \\
\text { controle }(\mathrm{n}=23) \\
\text { e um grupo caso } \\
(\mathrm{n}=43) \text {. A média } \\
\text { de idade materna } \\
\text { das saudáveis } \\
24 \pm 5 \text { e de mães } \\
\text { com PE foi } 25 \pm 5 .\end{array}$ & $\begin{array}{l}\text { o caso- } \\
\text { le }\end{array}$ & $\begin{array}{l}\text { Foram coletadas amostras } \\
\text { de cabelo retirados da } \\
\text { região púbica e sangue de } \\
\text { todas as participantes. Os } \\
\text { autores analisaram } \mathrm{As}, \mathrm{Ca} \text {, } \\
\mathrm{Cd}, \mathrm{Cr}, \mathrm{Co}, \mathrm{Mg}, \mathrm{Mn}, \mathrm{Fe}, \\
\mathrm{Cu}, \mathrm{Pb}, \mathrm{Se}, \mathrm{Ni}, \mathrm{Zn} \text {. }\end{array}$ & $\begin{array}{l}\text { As taxas séricas de } \mathrm{Zn} \text { se mostraram mais elevadas no grupo } \\
\text { caso }(0,16-253,4 \mathrm{mg} / \mathrm{L}) \text { em comparação com o grupo controle } \\
(0,2-48,4 \mathrm{mg} / \mathrm{L})(\mathrm{p}=0,01) \text {, pode-se notar que o } \mathrm{Zn} \text { estava } \\
\text { reduzido ao limite de detecção de instrumento para algumas } \\
\text { medições em ambos os grupos da pesquisa. }\end{array}$ \\
\hline $\begin{array}{l}\text { Wang et } \\
\text { al.(2018) }\end{array}$ & $\begin{array}{l}\text { Avaliar a associação entre } \\
\text { antioxidantes da ingestão } \\
\text { alimentar, como vitamina } \\
\mathrm{C} \text { e vitamina } \mathrm{E}, \mathrm{Zn}, \mathrm{Se}, \mathrm{Cu} \\
\text { e Mn, e o risco de } \\
\text { desenvolver distúrbios } \\
\text { hipertensivos gestacionais. }\end{array}$ & $\begin{array}{l}\text { Foram avaliadas } \\
10.228 \text { gestantes, } \\
\text { maiores de } 18 \\
\text { anos. }\end{array}$ & $\begin{array}{l}\text { caso- } \\
\text { le. }\end{array}$ & $\begin{array}{l}\text { Informações } \\
\text { características } \\
\text { demográficas, tabagismo, } \\
\text { consumo de álcool e chás, } \\
\text { idade gestacional, } \\
\text { histórico familiar de } \\
\text { hipertensão, hipertensão } \\
\text { na gravidez anterior, } \\
\text { histórico médico e e } \\
\text { reprodutivo, exposição } \\
\text { ocupacional e ambiente } \\
\text { residencial, atividade } \\
\text { física e situação alimentar. }\end{array}$ & $\begin{array}{l}\text { A ingestão de nutrientes antioxidantes na dieta, como } \mathrm{Zn} \text {, } \\
\text { durante a gravidez mostrou uma associação positiva } \\
\text { significativa com o desenvolvimento de distúrbios } \\
\text { hipertensivos da gestação, após ajuste para variáveis de } \\
\text { confusão (p < 0,0001). Após o ajuste para variáveis de } \\
\text { confusão e analisar ingestão alimentar de todos os nutrientes, } \\
\text { apenas Zn antes da gravidez mostrou uma positiva associação } \\
\text { com doença hipertensiva gestacional (p }<0,0001) \text {. Após o } \\
\text { ajuste para fatores de confusão e todos os antioxidantes, } \\
\text { apenas a ingestão de Zn durante a primeiro trimestre ou antes } \\
\text { da gravidez }(p=0,0054) \text {. }\end{array}$ \\
\hline $\begin{array}{l}\text { Enebe et } \\
\text { al. }(2020)\end{array}$ & $\begin{array}{l}\text { Determinar os níveis } \\
\text { séricos médios de alguns } \\
\text { de } \mathrm{Cu}, \mathrm{Zn}, \mathrm{Se}, \mathrm{Mg}, \mathrm{Mn} \text { na } \\
\text { fase pré-mulheres grávidas } \\
\text { eclâmpticas e comparar }\end{array}$ & $\begin{array}{lr}62 & \text { participantes } \\
(81 & \text { diagnosticas } \\
\text { com PE } & \text { e } 81 \\
\text { controle). } & \text { Idade } \\
\text { média de } & 29,5\end{array}$ & $\begin{array}{l}\text { Transversal } \\
\text { e analítico. }\end{array}$ & $\begin{array}{l}\text { Coleta de dados } \\
\text { socioeconômicos e análise } \\
\text { dos níveis de Se, } \mathrm{Zn}, \mathrm{Mn} \text {, } \\
\mathrm{Cu} \text { e Mg através de } \\
\text { amostras sanguíneas. }\end{array}$ & $\begin{array}{l}\text { A concentração sérica de Zn não variou entre os dois grupos, } \\
\text { no grupo de gestantes com PE sem detecção de gravidade a } \\
\text { média foi de } 0, .300(0, .179) \text { e no de gestantes com PE com } \\
\text { detecção de gravidade } 0, .453(0, .438)(\mathrm{p}>0,05) \text {. }\end{array}$ \\
\hline
\end{tabular}




\begin{tabular}{|c|c|c|c|c|c|}
\hline & com grávidas saudáveis. & $\begin{array}{l}\text { (idade entre } 18- \\
40 \text { anos). }\end{array}$ & & & \\
\hline $\begin{array}{l}\text { Mazloomi } \\
\text { et al. } \\
(2020)\end{array}$ & $\begin{array}{l}\text { Investigar a atividade da } \\
\text { NOS e da enzima } \\
\text { tiorredoxina redutase e a } \\
\text { quantidade de elementos } \\
\text { de Ca, } \mathrm{Zn} \text { e Se envolvidos } \\
\text { na função dessas enzimas } \\
\text { em mulheres com PE. }\end{array}$ & $\begin{array}{l}30 \text { mulheres } \\
\text { grávidas } \\
\text { saudáveis e } 30 \\
\text { mulheres com PE } \\
(\mathrm{n}=60) \text {, com idade } \\
\text { entre } 24 \text { e } 38 \\
\text { anos. }\end{array}$ & $\begin{array}{l}\text { Caso- } \\
\text { controle. }\end{array}$ & $\begin{array}{l}\text { Foram obtidos os dados } \\
\text { sobre idade, idade } \\
\text { gestacional, pressão } \\
\text { arterial, consumo de } \\
\text { minerais e suplementos na } \\
\text { gravidez. Foram coletados } \\
\text { peso e amostras de sangue. }\end{array}$ & $\begin{array}{l}\text { Zn sérico reduziu significativamente no grupo de gestantes } \\
\text { com PE quando comparado com o grupo controle saudável, a } \\
\text { média do grupo controle foi de } 43,86 \pm 105 \text { e a do grupo caso } \\
\text { foi de } 841,59 \pm 29,75(p=0,027) \text {. De acordo com a análise de } \\
\text { regressão, a variável e NOS no grupo controle em relação ao } \\
\text { Zn ( } p \text { valor }=0,049, r=0,344) \text { teve uma relação direta }\end{array}$ \\
\hline $\begin{array}{l}\text { Gul et al. } \\
(2021)\end{array}$ & $\begin{array}{l}\text { Investigar os níveis de Zn } \\
\text { e Cu no sangue de } \\
\text { mulheres grávidas com ou } \\
\text { sem PE. Além de avaliar o } \\
\text { status de tiol/dissulfeto em } \\
\text { dois grupos como um novo } \\
\text { marcador de estresse } \\
\text { oxidativo e também } \\
\text { procuramos uma possível } \\
\text { correlação entre os níveis } \\
\text { de tiol e os parâmetros } \\
\text { investigados. }\end{array}$ & $\begin{array}{l}88 \text { gestantes }(43 \\
\text { gestantes com PE } \\
\text { e } 45 \text { gestantes } \\
\text { saudáveis), com } \\
\text { idade entre } 18 \text { e } \\
45 \text { anos. }\end{array}$ & $\begin{array}{l}\text { Estudo } \\
\text { observacio- } \\
\text { nal caso- } \\
\text { controle. }\end{array}$ & $\begin{array}{l}\text { Foram coletadas amostras } \\
\text { de sangue para avaliar as } \\
\text { concentrações de } \mathrm{Zn} \text { e } \mathrm{Cu} \text {. }\end{array}$ & $\begin{array}{l}\text { O nível de } \mathrm{Zn} \text { encontra-se baixo em ambos os grupos, com } \\
\text { valor médio de } 80,3 \mu \mathrm{g} / \mathrm{dL} \text { nos controles e } 75,1 \mu \mathrm{g} / \mathrm{dL} \text { nas } \\
\text { pacientes. Não houve diferença significativa nos níveis de } \mathrm{Zn} \\
\text { na comparação entre os dois grupos }(\mathrm{p}>0,05) \text {. }\end{array}$ \\
\hline
\end{tabular}

Legenda: As: Arsênio; Ca: Cálcio; Cd: Cádmio; Co: Cobalto; CoQ10: Coenzima Q10; Cr: Cromo; Cu: Cobre; Fe: Ferro; Mg: Magnésio; Mn: Manganês; Ni: Níquel; PE: Pré-eclâmpsia; Pb: Chumbo; Se: Selênio; SOD: Superóxido Dismutase; Zn: Zinco. Fonte: Autores. 


\section{Discussão}

Os estudos incluídos nesta revisão, em sua maioria mostraram associação inversamente porporcional entre as concentrações séricas do Zn com a PE em mulheres gravidas. No entanto, a divergência nos achados ainda é marcante, alguns autores não encontraram associação estatisticamente significativa, tornando o tema em discussão ainda conflitante.

A PE é uma das complicações mais comuns durante a gestação, uma hipertensão induzida pela gravidez caracterizada por pressão arterial persistentemente elevada, mais de 140/90 mm Hg, e a que possui uma maior taxa de morbimortalidade e perinatal, apesar dos vários estudos sobre a PE, sua etiologia ainda não foi totalmente elucidada. Tal problema é $10 \%$ mais predominante em mulheres grávidas pela primeira vez, quando comparado com mulheres que passaram por gravidez anteriormente, além disso, a PE também atinge o recém-nascido (Ruiz, 2015; Farzin, 2012; Jamal, 2017).

Nesse sentido, a importância dos oligoelementos, particularmente o $\mathrm{Zn}$, na PE, relaciona-se ao fato que esse mineral está presente em diversas enzimas, sendo essencial no metabolismo, crescimento, desenvolvimento e reprodução. A sua deficiência é associada a complicações na gravidez, dentre elas, a PE, a concentração sérica desse mineral é reduzida em mulheres com PE em comparação com as saudáveis, indicando estar envolvido com a patogênese. Atualmente, tem sido observado um aumento na prevalência da PE de forma global, mas ainda é conflitante os relatos entre os oligoelementos e a sua patogênese (Farzin, 2012; Jamal, 2017; Al-Sakarneh, 2021).

Keshavarz et al. (2017) realizaram um estudo caso-controle com 200 participantes, no Irã, entre maio de 2014 e junho de 2015, onde foi demonstrado que os níveis plasmáticos de Zn diminuíram estatisticamente em mulheres grávidas com PE $(0,69 \pm 0,21)$ em comparação com o grupo controle $(0,87 \pm 0,30)$, os autores também avaliaram a atividade da enzima antioxidante superóxido dismutase (SOD), e observaram uma correlação inversa significativa entre os níveis de Zn e a atividade de SOD, que mostrou ter sido aumentada no grupo caso. Tal fato pode estar atrelado a respostas adaptativas causadas pelo nível de peroxidação lipídica aumentada nas integrantes do grupo caso, gerando o ataque de espécie reativas de oxigênio (EROs), que induzem o aumento compensatório dos níveis de enzimas antioxidantes.

Ademais, no estudo realizado por Mazloomi et al. (2020), também foi observado uma diminuição significativa dos níveis de Zn no grupo de gestantes com PE em comparação com o grupo controle. Bem como, pôde-se notar relação significativa e direta entre o Zn e a enzima óxido nítrico sintase (NOS) nos dois grupos. Sabe-se que os íons de Zn são capazes de se ligar a esta enzima, ativando-as, ou seja, a presença de íons de Zn pode aumentar a atividade da enzima NOS e, consequentemente, com a redução desses íons de Zn a atividade dessa enzima diminui (Forstermann, 2010; Lopez-Sepúlveda, 2008). Em um trabalho realizado por Shaikh et al. (2017), com 210 gestantes, verificaram a diminuição das concentrações de Zn no grupo de mulheres com PE em comparação com o grupo de gestantes normotensas.

Contraditório a esses achados, ao avaliar a ingestão de nutrientes antioxidantes, Oliveira et al. (2016) não encontraram diferenças significativas sobre as concentrações de $\mathrm{Zn}$ entre grupos controles e grupos de gestantes com PE, corroborando com os achados de Gul et al. (2021), onde as concentrações de Zn se mostraram baixas em ambos os grupos, não havendo diferença significativa, ao avaliar amostras sanguíneas. Isso pode estar relacionado a redução dos valores normais de Zn em mulheres saudáveis no terceiro trimestre de gestação. (Abbassi- Ghanavati, Greer \& Cunningham, 2009). Também é importante ressaltar que a necessidade de $\mathrm{Zn}$ tende a aumentar com a avanço da gestação, podendo estar associado a diminuição da capacidade de ligação do Zn e também aos efeitos dos níveis de estrogênio que podem causar estresse oxidativo (Ma; Shen \& Zhang, 2015).

Enebe et al. (2020) em um estudo transversal analítico com 162 gestantes notaram que, embora os níveis séricos médios de $\mathrm{Zn}$ nas mulheres com PE foram menores do que em mulheres normotensas, a significância não foi positiva ( $\mathrm{p}>$ 0,05). Corroborando também com os achados de Elmugabil et al. (2016), ao estudar os níveis séricos em 100 gestantes, não 
Research, Society and Development, v. 10, n. 8, e44810817451, 2021

(CC BY 4.0) | ISSN 2525-3409 | DOI: http://dx.doi.org/10.33448/rsd-v10i8.17451

encontraram nenhuma diferença significativa, concluíndo que os níveis séricos de Zn não estão associados com a PE.

Apesar disso, alguns estudos mostraram resultados contraditórios. Conforme Maduray et al. (2017), as gestantes com PE apresentaram concentração sérica de Zn maior do que gestantes saudáveis, esse trabalho contou com a participação de um grupo controle $(n=23)$ e um grupo caso $(n=43)$, no qual as taxas séricas de $\mathrm{Zn}$ se mostraram elevadas no grupo caso $(0,16$ 253,4 mg/L) em comparação com o grupo controle (0,2-48,4 mg / L). Dessa forma, os autores associaram esse aumento de $\mathrm{Zn}$ sérico como um fator etiológico em mulheres com hipertensão primária. No estudo, os autores relataram uma possível explicação, o excesso de Zn no organismo eleva as concentrações intracelulares $(r=-0,37, p=0,37)$. Observou-se que o desequilíbrio de ácido nítrico e as EROs apresentaram um papel importante na patogênese da PE, gerando disfunção endotelial.

Como alguns estudos propuseram, a deficiência de zinco pode estar associada a fisiopatologia da pré-eclâmpsia, embora alguns estudos demonstrem a inexistência dessa relação, a falta do zinco pode causar um maior estresse oxidativo aumentando a peroxidação lipídica pela diminuição dos antioxidantes de defesa dependentes do zinco, como a enzima SOD (Gul et al., 2021). Além da presente associação, a literatura mostrou que concentrações diminuídas de ZN em pacientes com pré-eclâmpsia podem ser resultado da diminuição dos níveis de albumina ocasionadas pela proteinúria característica da préeclâmpsia, uma vez que a albumina é o principal transportador de zinco no plasma (Kumera et al., 2015).

\section{Conclusão}

Concluiu-se com esta revisão sistemática que o mineral zinco desempenha um importante papel no desenvolvimento gestacional e fetal, e que a maioria dos estudos demonstrou uma associação positiva entre a carência de zinco e o surgimento da pré-eclâmpsia. Diante do exposto, fica evidente a importância da realização de investigações mais avançadas sobre a associação estudada nesse trabalho, assim como os mecanismos envolvidos, trazendo luz para os questionamentos acerca do assunto e servindo, também, para futuras ações de prevenção e tratamento desta patologia. Ao longo da produção desse artigo, pôde-se perceber a carência de estudos que abordem este tema, sendo assim, necessário realizar mais pesquisas que trabalhem essa temática.

\section{Referências}

Abbassi-Ghanavati, M., Greer, L. G., \& Cunningham, F. G. (2009). Gravidez e estudos de laboratório: uma tabela de referência para médicos. Obstetrics \& Gynecology, 114 (6), 1326-1331.

Chasapis, C. T., Loutsidou, A. C., Spiliopoulou, C. A., \& Stefanidou, M. E. (2012). Zinc and human health: an update. Archives of Toxicology. 86 (4), 521 534.

Cominetti, C., \& Cozzolino, S. M. F. (2020). Bases bioquímicas e fisiológicas da nutrição: nas diferentes fases da vida, na saúde e na doença. (2a ed.), Manole.

Elmugabil, A., Hamdan, H. Z., Elsheikh, A. E., Rayis, D. A., Adam, I., et al. (2016). Serum Calcium, Magnesium, Zinc and Copper Levels in Sudanese Women with Preeclampsia. PLoS ONE, 11 (12).

Enebe, J. T., Dim, C. C., Ugwu, E. O., Enebe, N. O., Meka, I. A., et al. (2020). Serum antioxidant micronutrient levels in pre-eclamptic pregnant women in Enugu, south-East Nigeria: a comparative cross sectional analytical study. Pregnancy and Childbirth, 20 (392).

Forstermann, U. (2010). Nitric oxide and oxidative stress in vascular disease. Pflügers Archiv: European Journal of Physiology, 459 (6), $923-939$.

Galvão, T. F., Pansani, T. S. A., \& Harrad, D. (2015). Principais itens para relatar Revisões sistemáticas e meta-análises: a recomendação PRISMA. Epidemiologia e serviços de saúde, 24 (2), 335-342.

Galvão, T. F., \& Pereira, M G. (2014). Revisões sistemáticas da literatura: passos para sua elaboração. Epidemiologia e serviços de saúde, 23 (1), $183-184$.

Grieger, J. A., \& Clifton, V. L. (2015). A review of the impact of dietary intakes in human pregnancy on infant birthweight. Nutrition Austrália, 7 (1), 153178.

Gul, A. Z., Atakul, N., Selek, S., Atamer, Y., Sankaya, U., et al. (2021). Maternal sérum levels of zinc, copper, and thiols in preeclampsia patients: a casecontrol study. Biological Trace Element Research. 
Research, Society and Development, v. 10, n. 8, e44810817451, 2021

(CC BY 4.0) | ISSN 2525-3409 | DOI: http://dx.doi.org/10.33448/rsd-v10i8.17451

Jamal, B., Shaikh, F., \& Memon, M. Y. (2017). To Determine the Effects of Copper, Zinc and Magnesium in Patients with Pre-Eclampsia. Journal of the Liaquat University of Medical and Health Sciences, 16 (01).

Keshavarz, P., \& Nobakht, M. G. B. F., Mirhafez, S. R., Nematy, M., Azimi-Nezhad, M., et al. (2017) Alterations in Lipid Profile, Zinc and Copper Levels and Superoxide Dismutase Activities in Normal Pregnancy and Preeclampsia. The American Journal of the Medical Sciences, 353 (6), $552-558$.

Kumera, G., et al (2015) Prevalência de deficiência de zinco e suaassociação com dieta, albumina sérica e parasitas intestinaisinfecção entre mulheres grávidas atendidas no pré-natal no Hospital da Universidade de Gondar, Gondar, Noroeste da Etiópia. BMC Nutrition, 1 (3).

Latif, A. A., Manzoor, F., Mushtaq, S., Noureen, T., Fatima, S., et al. (2016). Serum Concentration of Zinc in Healthy Pregnant Women Versus Pre-Eclamptic Pregnant Women: A case Control Study in Lahore, Pakistan. Medical Forum Monthly, 27 (1).

Lopez-Sepúlveda, R., et al. (2008). Wine polyphenols improve endothelial function in large vessels of female spontaneously hypertensive rats. Hypertension, $51(4), 1088-1095$.

Ma, Y., Shen, X., \& Zhang, D. (2015). The Relationship between Serum Zinc Level and Preeclampsia: A Meta-Analysis. Nutrients, 7 (9), 7806-20.

Maduraya, .K, et al. (2017). Elemental analysis of serum and hair from pre-eclamptic South African women. Journal of Trace Elements in Medicine and Biology.

Mazloomi, S., Khodadadi, I., Aimohammadi, S., \& Shafiee, G. (2021) Correlation of thioredoxin redutase (TrxR) and nitric oxide synthase (NOS) activities with sérum trace elements in preeclampsia. Clinical and Experimental Hypertension, 43 (2), 120-124.

Mistry, H. D., Kurlack, L. O., \& Young, S. D. (2014). Maternal selenium, copper and zinc concentrations in pregnancy associated with small-for-gestationalage infants. Maternal and Child Nutrition, 10 (13), 327-334.

Oliveira, A. C. M., et al. (2016). Intake of antioxidant nutrients and coefficients of variation in pregnant women with preeclampsia. Revista Portuguesa de Cardiologia, 35 (9), 469-476.

Prado, M. R., et al. (2015). Prevalence of vitamin D deficiency and associated factors in women and newborns in the immediate postpartum period. Revista Paulista de Pediatria, 33 (1), 287-294.

Ruiz, M. T., et al. (2015). Associação entre síndromes hipertensivas e hemorragia pós-parto. Revista Gaúcha de Enfermagem, 36, 55-61.

Sanchez-Aranguera, L. C., et al. (2014). Disfunção endotelial e pré-eclâmpsia: papel do estresse oxidativo. Frontiers in Physiology, 5, 1-11.

Santos, C. M. C., et al. (2007). A estratégia PICOS para a construção a pergunta de pesquisa e busca de evidências. Rev. Latino-Am. Enfermagem, 15 (3).

Shaikh, F., Shah, T., Ansari, S., \& Dahri, S. (2017). To determine the role of co-enzyme Q10 and trace elements in patient with pre-eclampsia - a cross sectional study in Hyderabad. Journal of the Liaquat University of Medical and Health Sciences, 16 (2), 86-92.

Thomas, D. W., et al. (2013). Guideline for the laboratory diagnosis of functional iron deficiency. British Journal of Haematology. 161 (5), 639-648.

Wang, Z., et al. (2018). The association between dietary vitamin C/E and gestational hypertensive disorder: a case-control study. J. Nutr. Sci. Vitaminol, 64, 454-465.

Yasuda, H., \& Tsutsui, T. (2016). Infants and elderlies are susceptible to zinc deficiency. Scientific Reports, 6 (21850). 